\title{
Estimation of expected utility gained from the helmet law in Taiwan by quality-adjusted survival time
}

\author{
Jau-Yih Tsauo a, Jing-Shiang Hwang ${ }^{\mathrm{b}}$, Wen-Ta Chiu ${ }^{\mathrm{c}}$, Ching-Chang Hung ${ }^{\mathrm{d}}$, \\ Jung-Der Wang ${ }^{\mathrm{a}, *}$ \\ ${ }^{a}$ Center for Research of Environmental and Occupational Diseases, Institute of Occupational Medicine and Industrial Hygiene, \\ National Taiwan University College of Public Health, No. 1, Section 1, Jen Ai Road, Taipei 10016, Taiwan \\ ${ }^{\mathrm{b}}$ Institute of Statistical Science, Academia Sinica, Taipei 115, Taiwan \\ c Taipei Medical College Hospital, Taipei, Taiwan \\ d National Taiwan University Medical College, Department of Surgery, Taipei, Taiwan
}

Received 10 April 1998; received in revised form 10 November 1998; accepted 4 December 1998

\begin{abstract}
The objective of this study was to empirically estimate the expected utility gained from the implementation of the 1997 helmet law in Taiwan by using quality-adjusted survival time (QAST). We randomly selected 400 out of 8221 registered cases of head injury and successfully interviewed 99 cases with the index of health-related quality of life (IHRQ). The function of IHRQ was then multiplied with the corresponding survival function to obtain the QAST for head injury. The total utility gained from the helmet law in Taipei within 1 year was estimated by multiplying the expected loss of utility per patient with the number of prevented cases. The results showed that after 80 months of follow-up, the QAST of the injured population was 66.3 quality-adjusted life-months (QALMs), while that of the reference population was 78.7 QALMs. We extrapolated the QAST for total life expectancy by simulating the survival of head injury cases using the life table data from the general population. The life-long utility loss of a head injury case was found to be 4.8 quality-adjusted life-years (QALY). The number of prevented cases during the first year of enforcement of the helmet law was estimated to be 1300 cases in Taipei, which amounted to 6240 QALYs gained. For lack of data, we were unable to calculate the possible gain from helmet on reduction of severity among nonfatal cases with head injury, and the estimation was only a lower bound. We concluded that the QAST approach is a feasible approach applicable to health policy decision-making, especially in cost-utility analysis. (C) 1999 Elsevier Science Ltd. All rights reserved.
\end{abstract}

Keywords: Quality-adjusted survival time; Cost-utility analysis; Head injury

\section{Introduction}

In many countries, the cost of health services has been growing rapidly for the last several decades (Sorkin, 1992). Although many remedies to control health care spending have been proposed, controversy exists on the long-term effectiveness of simply focusing on the supply side or changing the output of health services in the health care system (Patrick and Erickson, 1993).

Cost-utility analysis (CUA), a common method for evaluating health care programs (Drummond et al.,

\footnotetext{
* Corresponding author. Tel.: + 886-223-562224; fax: + 886-23224660; e-mail: jdwang@ha.mc.ntu.edu.tw.
}

1987; Robinson, 1993), is used to measure the cost per unit of utility gained. Utility refers to the value or worth of a specific level of health status and can be measured by the preferences of individuals or society for any particular set of health outcomes. Current methods of estimating the utility of health (Morrow and Bryant, 1995) include the following approaches: the Ghana Health Assessment Team (GHAT) approach (Ghana Health Assessment Project Team, 1981), the quality-adjusted life-year (QALY) approach (Torrance, 1986), the disability-adjusted life-year (DALY) approach of the World Development Report (Murray, 1994), the quality-adjusted survival estimation with partitioned survival approach (Glasziou et al., 1990), and the quality-adjusted survival time (QAST) approach (Hwang et al., 1996). 
In general, the GHAT approach measures the total utility remaining when an illness occurs, by assigning a specific quality of life value to patients and estimating their life expectancy from the general population's life table. However, most chronic diseases, e.g. stroke or diabetes mellitus, will usually shorten patients' life spans and progressively lower their HRQL (health-related quality of life) with time. As a result, the GHAT calculation may not be an accurate estimate of utility loss.

QALY is arrived at in each case by adjusting the length of time affected through the health outcome by the utility value (on a scale of $0-1$ ) of the resulting level of health status. The usual QALY approach may be accurate and applicable for clinical trials with a short termination point. Yet, it is usually not feasible to continue measurements of HRQL throughout a patient's survival period if it is too long, e.g. more than 10 years. The DALY approach avoids the above pitfalls by generating both a survival function and a disabilityadjusted utility function over time from a group of experts. However, a democratic society may question the direct use of DALY in resource allocation because the people of a community must rely on an authority's opinion, rather than their own, to measure survival function and a disability-adjusted utility function over time (Gold et al., 1996). Glasziou et al. proposed the partitioned survival approach to handle this problem (Glasziou et al., 1990). While their model treated the progress of illness as several discrete states, which is applicable for certain types of cancer, it may not be equally applied to other chronic diseases, of which disease states are often highly variable.

Hwang et al. proposed the QAST approach (Hwang et al., 1996) which simultaneously considers both survival and HRQL values over time directly from the patients. Thus, QAST seems suitable as a universal approach to evaluating health care. However, the QAST methodology has not yet been demonstrated in an empirical study.

Head injury has been a significant public health problem in Taiwan, occurring at a rate of about 182 per 100000 population per year in Taipei and 304 per 100000 population per year in Hualien, according to a comprehensive registration program, which included all cases came to the emergency rooms (Chiu et al., 1996). A sample was obtained from this registration program, and the survival function and HRQL for cases of head injury were calculated. Then, the average utility loss per case of head injury was estimated using the QAST method.

A helmet law was implemented in Taiwan on January, 1997 and enforced beginning on June 1, 1997. The proportion of motorcyclists with no helmet decreased dramatically, and the occurrence of severe head injury decreased thereafter. The purpose of this study was to calculate the number of head injuries prevented and the QALYs saved from the enforcement of the helmet law by using the QAST approach.

\section{Subjects and method}

\subsection{Subjects}

From July 1, 1989 to June 30, 1994, the Head and Spinal Cord Research Group reported that there were 8221 head injury cases from motorcycle accidents in Taipei (Chiu et al., 1996). 80 cases from each year were randomly selected, for a total of 400 cases. Of these, 391 had provided a telephone number when they were first admitted to the hospital. After matching the death certificate computer files provided by the Department of Health, 44 deaths were found out of the 400 cases. A total of 242 cases had moved to another addresses or had changed their telephone numbers, and six cases refused to be interviewed. Finally, in the time period between December, 1995 and February, 1996, 99 cases were successfully interviewed by telephone.

\subsection{Assessment of the HRQL of head injury cases}

The subject-interview was performed using a structured questionnaire consisting of background data and measurements of HRQL. Background data included age, sex, education, occupation and date of injury. HRQL was measured according to the index of healthrelated quality of life (IHQL), a 3-dimensional classification system (Rosser et al., 1992) translated to fit Taiwanese society. Briefly, each case was asked about his recent health profiles regarding dimensions of disability, discomfort and emotional condition. There are eight levels ranged from no physical disability to unconsciousness in disability dimension, five levels ranged from no pain to agonising pain in discomfort dimension, and five levels ranged from no distress to extremely depressed in emotional dimension. Valuation for the composite health status of each case was obtained from a utility valuation table proposed by Rosser et al. 1992. If the patients could also clearly recall their health conditions at the end of each year after head injury (not only the health condition at the time of interview), such IHQL profiles were also obtained for the years followed injury. A total of 89 out of 99 cases could recall their health condition upon discharge from the hospital, which generally happened within one or two months after the injury. Some of them could even recall their condition in the years that followed. Therefore, each case could contribute one or more data points in this study and a total of 288 data points were provided and shown on Fig. 1. 
A kernel-type smoother (Watson, 1966) was then applied to the 288 data points to estimate the mean quality of life at months 1 through to 80 . The mean of the nearest 15 sample points ( $5 \%$ of the 288 points) in each month was used to represent the mean HRQL of that month.

For test-retest reliability, 20 cases were randomly selected and called a second time 11-13 months after their first interview in the interval of December, 1995 to February, 1996. In their second interview during January, 1997, they were asked to recall their health conditions during the first interview.

\section{3. $H R Q L$ of the reference population}

The reference HRQL of people younger than 60 years of age was assumed to be one. For people age 60 and older, a national HRQL survey of senior citizens in Taiwan was conducted by the Taiwan Provincial Institute of Family Planning (TPIFP) in 1989, in which they randomly interviewed 4049 people, age 60 and over, to obtain detailed information on their HRQL (Taiwan Provincial Institute of Family Planning, 1989). Scales of the 3-dimensions required for IHQL (disability, discomfort and emotional condition) were abstracted to obtain the HRQL value.

\subsection{Survival function of head injury cases}

Since 1930, Taiwan has had a comprehensive system of national death certification. Moreover, according to the Ministry of Interior Affairs, the average emigration rates in Taiwan were $0.12-0.23 \%$ during $1989-1994$ (Ministry of the Interior, 1994). To generate the survival function, 1989-1995 computerized death certificate files from the Department of Health were matched with the 400 randomly sampled head injury cases by using their individual identification (ID) numbers. It was further assumed that people were highly unlikely to seek care outside of Taiwan because of the highly qualified neurosurgeons and rehabilitation personnel available in the country. Even if some had sought care and died elsewhere, their bodies would have likely been returned to Taiwan for burial, and the death registration agency would have been notified. In total, 44 decedents were obtained, and their survival function for head injury could be accurately estimated for the 80 months of follow-up (the end of 1995).

For currently surviving cases, it was assumed that they had the same life expectancy as the reference population of the same age and gender. Instead of directly taking the mean life expectancy for each case, we applied a Monte Carlo approach (Rubinstein, 1981) to account for possible random variation. The following method was used to estimate their expected survival function after censorship. First, a survival curve was created for each case according to the person's age and gender and the vital statistics of Taiwan (Ministry of the Interior, 1989-1993). Second, 356 random numbers were generated from a uniform distribution to represent the survival probability for each surviving case. The survival probabilities were then used to find the corre-

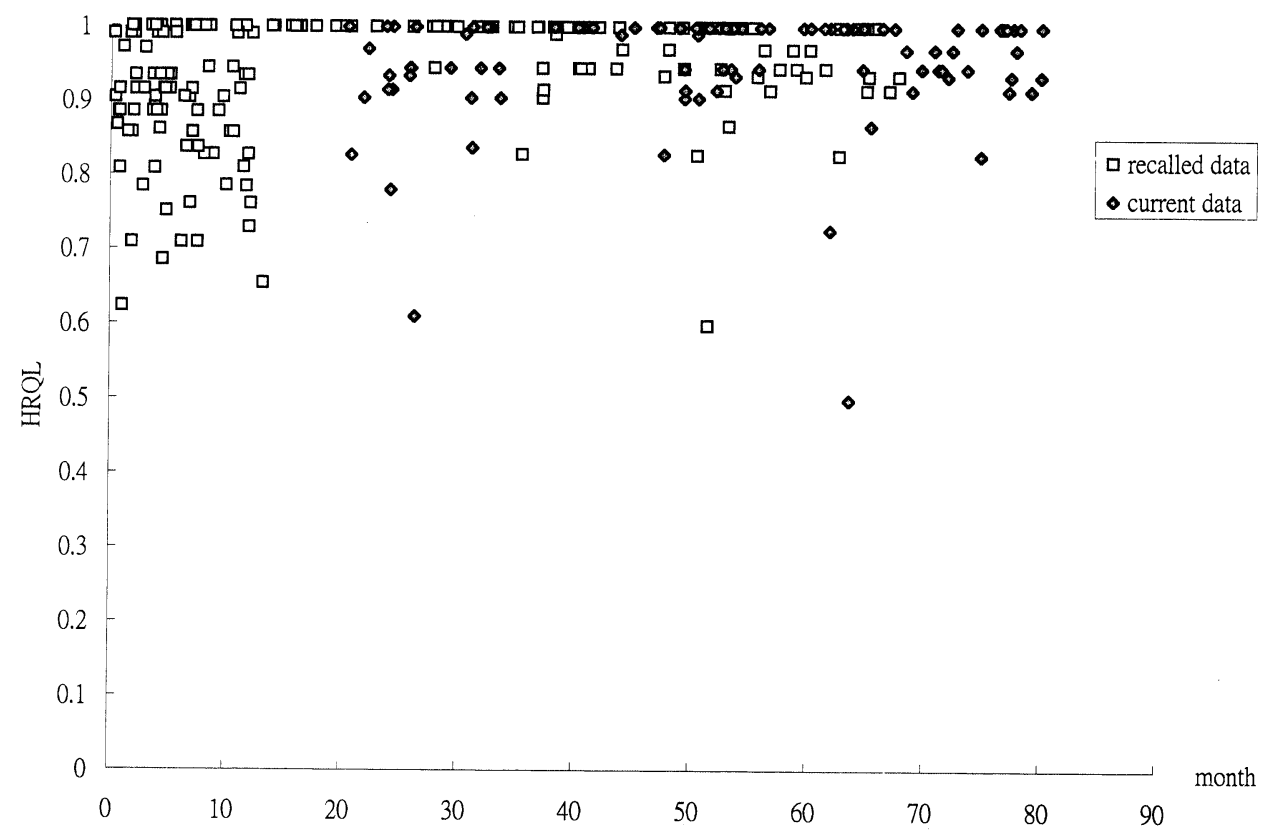

Fig. 1. Scatter plot of HRQL (health-related quality of life) data by months of follow-up: current data indicated the HRQL at the time of interview, while recalled data were obtained by enquiring from subjects' memory. 
sponding life expectancies on the survival curves created in the first step for each surviving case. Thus, 44 life spans were obtained from the decedents and 356 simulated life expectancies from the living cases. The survival function was then estimated using these 400 survival times by the Kaplan-Meier method (Lee, 1992) with S-plus software package (Stat Sci Division, 1995).

\subsection{Survival function of the reference population}

To obtain the expected survival function of the reference population, people of the same age and sex as in the random sample of 400 were used in the estimation of the survival function. A total of 400 random numbers were generated from a uniform distribution, and 400 life expectancies were then calculated from each survival curve. This pool of life spans was used in the estimation of the survival function for the reference population.

\subsection{Expected quality-adjusted survival time (QAST) of head injury and reference population}

The expected QAST of the two populations can be calculated by multiplying the HRQL into the survival function as in the following equations (Hwang et al., 1996). QAST of a period (80 months in this study) for a person is summed up by multiplying the QS of each interval and the length of the interval (Eq. (1)).

$\mathrm{QAST}=\sum_{i=0}^{80} \mathrm{QS}_{i} \times\left(t_{i+1}-t_{i}\right)$

where, QAST, quality-adjusted survival time; $\mathrm{QS}_{i}$, quality-adjusted survival time in the time interval $\left(t_{i}, t_{i+1}\right)$, which is equal to one month in this paper.

$$
\begin{aligned}
\mathrm{QS}_{i}= & {\left[S\left(t_{i}\right)-S\left(t_{i+1}\right)\right] / 2 \times\left[\mathrm{qol}\left(t_{i}\right)+\mathrm{qol}\left(t_{i+1}\right)\right] / 2 } \\
& +S\left(t_{i+1}\right) \times\left[\mathrm{qol}\left(t_{i}\right)+\mathrm{qol}\left(t_{i+1}\right)\right] / 2
\end{aligned}
$$

where, $S\left(t_{\mathrm{i}}\right)$, survival probability at time $t_{i} ; \operatorname{qol}\left(t_{i}\right)$, mean health-related quality of life at time $t_{i}$.

The first term of Eq. (2) corresponds to the qualityadjusted survival portion contributed by patients who died in $\left(t_{i}, t_{i+1}\right)$, and the second term is derived from patients who still survive at $t_{i+1}$. To calculate the QS in an interval $t_{i}$ ( 1 month in this study), we need the survival probabilities and the mean HRQLs from patients who died in interval $t_{i}$ and patients who still survive at the next interval $\left(t_{i+1}\right)$. Multiplying the HRQL with the survival probability for these two groups individually and then summed them up, we obtained the QS in this interval. Finally, we added up all $\mathrm{QS}_{i}$ for each interval $i$ to get the QAST.

A bootstrap method was performed to obtain an estimate of the variance of QAST (Hwang et al., 1996).
The bootstrap was implemented by conducting repeated sampling 50 times.

To calculate the life-long loss of QAST from head injury, the survival function of the simulated life spans of the surviving head injury cases and the 400 reference population cases (which were estimated as described earlier) was used. For the HRQL of head injury patients, the interviewed data for the first 7 years after the onset of head injury was used, and 0.963 (which was the mean HRQL of the 7th year) was then applied for the seventh and subsequent years, until age 60. A survey, conducted by the TPIFP (Taiwan Provincial Institute of Family Planning, 1989, measuring the HRQL of the general population, age 60 and over, was used to estimate the HRQL when cases reached the age of 60 and over. The difference between the QAST of the surviving head injury cases and the reference population was the expected loss of QAST due to head injury.

The number of cases of head injury can be derived from the equations in the Appendix A, which can be simplified as follows for a period of 1 year:

$N_{t} \times\left(I R_{1} \times P e_{1}+I R_{0} \times P e_{0}\right)$

where, $N_{t}$, average size of population at risk during year $t ; I R_{1}$, incidence rate of head injury for motorcyclists with no helmet; $P e_{1}$, proportion of motorcyclists with no helmet; $I R_{0}$, incidence rate of head injury for helmeted motorcyclists; $P e_{0}$, proportion of helmeted motorcyclists.

With the enforcement of the helmet law, the proportion of helmeted motorcyclists dramatically increased. After calculating the expected number of prevented cases according to the empirical data and the above equation, the utility gained could be estimated by multiplying the expected loss of QAST of one case with the total prevented number of cases.

\section{Results}

The demographic characteristics of the 8221 registered cases, the 400 randomly sampled cases and the 99 interviewed cases are summarized for comparison in Table 1. There were no statistically significant differences in distribution among these three groups in terms of age, gender and severity of injury. For cases of head injury, the probability of survival was 0.9225 during the first month and 0.895 during the first year. The reference population suffered few losses until the beginning of the second year. The survival curves of these two groups are plotted in Fig. 2. Log-rank test showed a statistically significant difference $(P<0.01)$ between these two curves, for both 80 months of follow-up and life-long simulation.

A scatter plot of the 288 data points of HRQL was shown in Fig. 1, which indicated a similar distribution 
Table 1

Comparison of frequency distributions on age, sex and severity among total registered cases $\left(n_{1}\right)$, sampled cases $\left(n_{2}\right)$ and cases successfully interviewed $\left(n_{3}\right)$



\footnotetext{
${ }^{\mathrm{a}} \chi_{(6)}^{2}=7.742, P=0.258$.

${ }^{\mathrm{b}} \chi_{(2)}^{2}=0.974, P=0.615$.

${ }^{\mathrm{c}} \chi_{(8)}^{2}=1.994, P=0.737$.
}

between the 99 current and 189 recalled points. The mean HRQL of head injury cases in the first year was 0.908 , lower than the mean of the subsequent years, 0.958 . The number of health states at each dimension was shown in Table 2. The mean HRQL of the head injury cases by the GOS (Glascow outcome scale) and time since injury was summarized in Table 3 . In the reference population, however, the corresponding HRQL was assumed to be one for the period under 60 years of age, and dependent on the surveyed data for the period at and over 60 years. The HRQL from month 1 to 80 of the two groups are shown in Fig. 3.

After combining the HRQL and survival functions, the quality-adjusted survival time (QAST) for an average person in the two groups was obtained, as shown in Fig. 4. The area below the curve is the estimation of expected $Q A S T$ of an average person. The expected QAST of the reference population during the 80 months was found to be 78.7 quality-adjusted life months (QALM) and that of the head injury cases was 66.3 QALMs. The shadowed area in Fig. 4 indicates the average loss of expected QAST of a head injury case compared with the reference population, which was calculated to be $78.7-66.3=12.4$ QALMs, over the period of 80 months after injury. The variance of expected QAST estimated with the bootstrap method was 1.3 QALMs. The results showed that there was a significant difference between the expected QAST of head injury cases and the reference population.
When the calculation was extended to that of life expectancy, the average loss of expected QAST of one case was 4.8 QALYs.

The number of head injury cases among motorcyclists, 1 year prior to the helmet law, was estimated to be 2541 (Table 4). Assuming that after the enforcement of the helmet law, the proportion of motorcyclists with no helmets dropped from $88 \%$ (Tsai et al., 1996) to 5\%, as reported by the traffic police department of Taipei, and the proportion of full face vs. partial coverage helmets did not change with implementation of the law; then the expected number of head injuries would be 1241 after one year of law enforcement. And the number of prevented cases would be 1300 . Thus, the total utility gained from the execution of the helmet law in 1 year would be $6240(=4.8 \times 1300)$ QALYs.

\section{Discussion}

From our estimation, the average lifelong loss in a head injury case was 4.8 QALYs, which was higher than the 4.6 QALYs computed from GHAT (Ghana Health Assessment Project Team, 1981). The difference between these two methods may be low for head injury or other acute diseases which do not affect the survival after acute stage. Our follow-up for 80 months also showed a similar survival function as the general population 1 year after injury. For other diseases, such as 


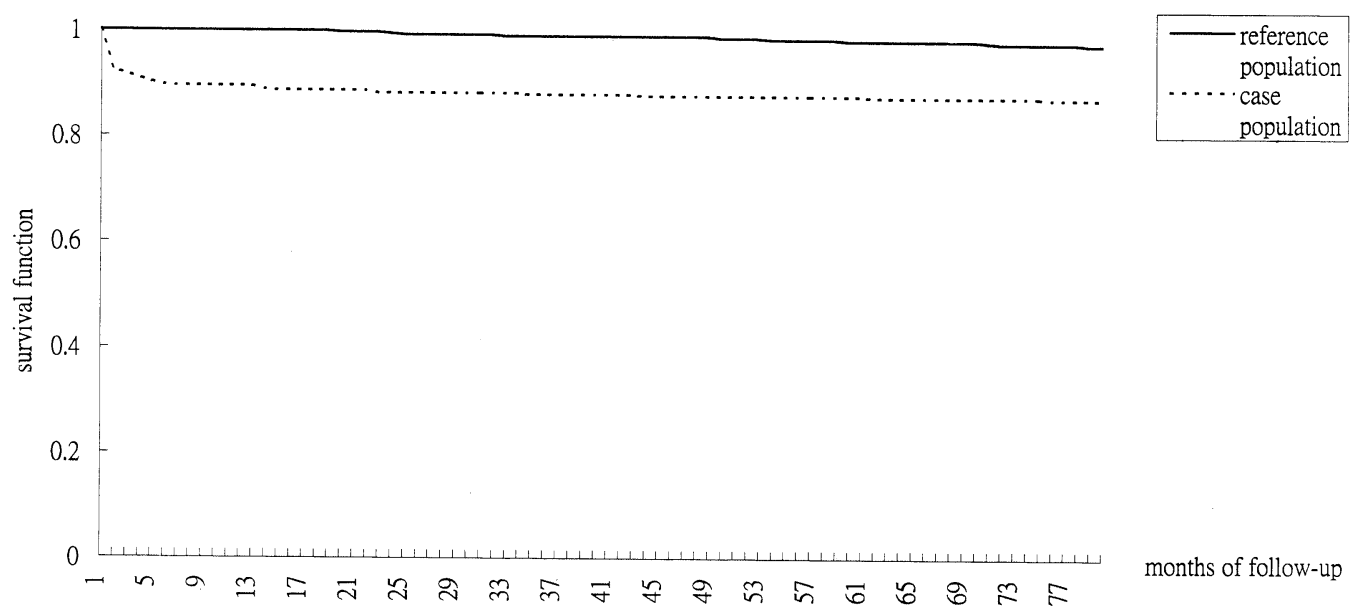

Fig. 2. The survival function of the head injury cases and the reference population in the 80 months after onset.

stroke or diabetes mellitus, with sustained mortality during the follow-up period, the GHAT estimation might not be so close to the QAST estimation.

Although using expected QAST to estimate QALY may be a more general solution to the measurement of utility of health, some components in its empirical application deserve clarification.

\subsection{Follow-up period}

The follow-up interval must be long enough to generate a complete survival curve for head injury cases. Since a large proportion of head injury cases with complete recovery and none or minimal sequela (91.8\%) may have the same life expectancy as a non-injured person of the same age, we created a survival function corresponding to the age and sex of each sampled case of head injury. Actually, 32 of 44 cases died within 1 month after injury, and 10 more died within 1 year. As a result, the assumption that cases, surviving past the first year, would have a normal life expectancy seemed acceptable. In the future, similar calculations can be tried for a reference population chosen deliberately, if there are more than one set of survival data available. While the above assumption may be tenable for an acute disease, such as head injury; chronic diseases with possibly high rates of censorship, such as diabetes mellitus, hypertension, etc., may require a longer period of follow-up to accurately estimate the survival function for QAST.

\subsection{The HRQL measurement}

The validity and reliability of the HRQL measurement must be concerned. The mean HRQL was 0.969 from the first interview and 0.979 from the second interview. The correlation coefficient was 0.736 , with $P=0.0002$. The within subject reliability $11-13$ months apart seemed acceptable. However, direct utility measurement (e.g. standard gambling) was not conducted in our survey owing to limited time. The original values of IHQL for the various health states were obtained from health professionals in the UK using the standard gamble method of one year's duration (Rosser et al., 1992). Thus, in this study, we presumed that health professionals in different countries share a similar preference for different health states. However, as people with different cultures may have different preferences, the valuation system for Taiwanese culture should be established in the future for a more accurate estimation.

Table 2

Number of cases at each level in each dimension of Rosser's scale

\begin{tabular}{lc}
\hline Dimension and levels & No. of cases $(\%)$ \\
\hline Disabilty & \\
No physical disability & $186(64.6)$ \\
Slight social disability & $59(20.5)$ \\
Slight physical disability & $29(10.1)$ \\
Can get around house and do light physical & $8(2.8)$ \\
$\quad$ work & \\
Difficulty in getting around house & $1(0.3)$ \\
Confined to a chair & $2(0.7)$ \\
Confined to bed & $3(1.0)$ \\
Unconscious & $0(0)$ \\
Discomfort & \\
No pain & $158(54.9)$ \\
Slight pain & $99(34.4)$ \\
Moderate pain & $29(10.7)$ \\
Severe pain & $2(0.7)$ \\
Agonising pain & $0(0)$ \\
Distress & \\
No distress & $219(76.0)$ \\
Slight distress & $51(17.7)$ \\
Moderate distress & $14(4.9)$ \\
Severe distress & $4(1.4)$ \\
Extremely depressed & $0(0)$ \\
& \\
&
\end{tabular}

a The 99 cases provided these 288 data points of health states. 
Table 3

The mean HRQL (health-related quality of life) of the cases of head injury by the time since injury and in each GOS (Glascow outcome scale) level

\begin{tabular}{llll}
\hline Years after injury & GOS $=3$ & GOS $=4$ & GOS $=5$ \\
\hline 1 & 0.754 & & 0.926 \\
2 & & 0.890 & 0.931 \\
3 & & 1.000 & 0.979 \\
4 & & 0.962 & 0.965 \\
5 & 0.668 & 0.908 & 0.931 \\
6 & & 0.978 & 0.943 \\
7 & & & 0.906 \\
\hline
\end{tabular}

In fact, quite a few methods have been developed to measure HRQL, including generic and disease-specific forms. Examples of generic forms include the Nottingham health profile (Hunt et al., 1986), the sickness impact profile (Bergner et al., 1981), Euroqol (Euroqol group, 1990), the short-form 36 health survey (Ware and Sherbourne, 1992), quality of well-being scale (Kaplan and Bush, 1982) and the IHQL (Rosser et al., 1992). Examples of injury-specific forms include the functional capacity index (MacKenzie et al., 1996), the injury impairment scale (Miller, 1993), etc. The IHQL was used in this study because of the conceptual clarity in using three major dimensions and the corresponding $0-1$ utility score which can be used in the estimation of QAST. In the future, different scales (including the scale obtained by the utility measurement) of HRQL might be used to perform a sensitivity analysis of utility estimated by QAST.

\subsection{Representativeness of the sample}

A total 242 sample cases moved or changed their phone numbers and lost to be interviewed. This may lead to a bias if the characteristics of the non-respon- dents differed significantly from the respondents. However, excluding the non-contact sample cases, the response rate in our study was over $94 \%$ (i.e. 99/105). Besides, the distributions for severity of injury and other demographic factors (e.g. age and sex) showed no significant differences among the total sampled cases and the cases successfully interviewed (Table 1). Thus, we believed that there was minimal bias in our sample. If the cases lost in follow-up had suffered from a more severe head injury, the HRQL of cases interviewed might have been overestimated and the loss of QAST might have been underestimated.

\subsection{Recall bias}

Recall bias is another concern in this study. Many cases provided three or more data sets of HRQL during different years. The utility scores for the first year after injury were stratified by recall time since injury. There was no significant difference in the utility score of HRQL for the first year between cases who were injured recently and those injured for more than 2 year $\left(t_{(\text {d.f. }=89)}=1.24, P=0.33\right)$. Moreover, if the recalled data were discarded and the QAST were recalculated with only 99 data points, then the result would be 65.7 QALMs, which is within the $95 \%$ confidence interval of $66.3 \pm 1.1$ QALMs calculated from the 288 data points. Therefore, data recalled from over 1 year showed a slight overestimation compared with current year recollections, which indicated that patients may more likely forget their suffering after more than 1 year.

\subsection{Underestimation}

Wearing a helmet could reduce mortality of head injury and modify the injury patterns and severity of the surviving cases (US Department of Transportation,

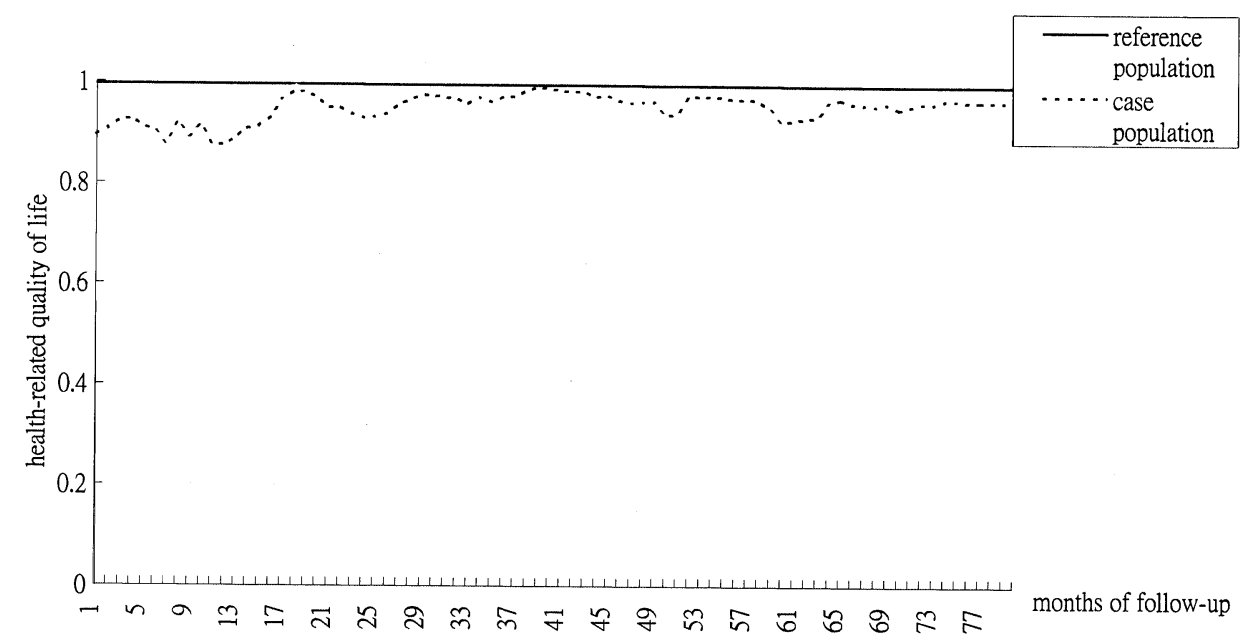

Fig. 3. The health-related quality of life of the head injury cases and the reference population in the 80 months after onset. 




Fig. 4. The quality adjusted survival time of the head injury cases and the reference population in the 80 months after onset.

1998, National Highway Traffic Safety Administration). From the Taipei registry of head injury, information was obtained on the helmet wearing states of 1360 out of 8221 registered subjects, which is summarized on Table 5. All 28 subjects who wore helmets experienced full recovery, a Glascow outcome scale of five, and a shorter length of stay, as compared with 1322 subjects who did not wear helmets. Thus, only counting the number of prevented cases in this study might have underestimated the impact of helmet wearing because the helmeted cases had a significantly less severe course $(P<0.05)$. In the future, we hope to improve the quality of information collected by the registry, and we hope to determine the QOL of cases with helmet versus no helmet. Then, a more comprehensive estimate of the total benefit gained from a preventive program may be obtained using Eq. (7) in the Appendix A.

\subsection{The assumptions and limitations of utilitarian approach}

The assumptions and limitations of the utilitarian approach in this paper need to be repeatedly mentioned so that it will be applied with adequate precaution. As the QALY obtained from the QAST method inherently depends on the age and life expectancy of each specific population, there is potential for discrimination against senior citizens (LaPuma and Laqlor, 1990). To avoid age discrimination, we propose that health policy decisions, which are concerned with resource allocation across age and disabilities, be based on pure mortality indices first. Then, consider QAST as a supplement within the same age stratum, with each stratum spanning 10-15 years (Hwang et al., 1996).

In conclusion, the application of this model to head injury cases empirically demonstrates the usefulness of the QAST method in estimating utility for the CUA (cost utility analysis) of an acute injury or disease. However, it still has not comprehensively solved some problems encountered with chronic diseases, such as high censorship rates, because of inadequate follow-up time. More empirical studies are needed to test the scope of its use. Yet, even with the above limitations, the QAST model of utility estimation should still be explored for use in risk assessment, health policy evaluation and clinical decision-making.

\section{Acknowledgements}

This study was supported by contract grant No. DOH87-HR-504, from the National Health Research Institute, Department of Health, Executive Yuan, ROC.

\section{Appendix A}

To calculate the expected number of prevented cases resulting from a prevention program, one must first assume that the etiologic fraction is estimated by the excess fraction from epidemiological studies. Then, let us first consider the simplest case of a prevention program, involving the elimination of one particular exposure which can result in one specific disease $d_{i}$.

Suppose that the activity of the prevention program begins at time $t_{0}$, and the proportion of exposure also begins to decrease at the same time because of this activity. Moreover, assume that the prevention program completes at time $t_{1}$, and the reduction of proportion of exposure also becomes stabilized after $t_{1}$. Then, after a period of induction $I_{d_{i}}$, the effect of prevention 
appears and the number of incidence cases begins to decrease, which is a function of the decreased proportion of exposure. The formula is derived as follows:

Let $N(t)$ denote the number of people in the population at time $t$ and $P e(t)$ represent the proportion of exposed population. Both are a function of time $t$. Let $I R_{1 d_{i}}$ be the incidence rate of the exposed population and $I R_{0 d_{i}}$ for the non-exposed population; both are assumed to be constant (no change in other determinants) over the observation time. If the prevention program starts at time $t_{0}$ and finishes at $t_{1}$, then the corresponding change of the exposure proportion during $t_{0}-t_{1}$ is $\mathrm{Pe}\left(t_{1}\right)-\mathrm{Pe}\left(t_{0}\right)$. Assume that the induction time $\left(I_{d_{i}}\right)$ for producing a preventive effect (reversing the process of developing the disease) is relatively short, so that the competing risk of dying from other diseases in the population is small and will not change $I R_{1 d}$ or $I R_{0 d_{i}}$ significantly during the study period. Thus, after time $I_{d}$, the number of prevented cases is the number of incidence cases that would have occurred under the prevention program, as seen in the following equations:

$$
\begin{aligned}
& \int_{t_{0}+I_{d_{i}}}^{t_{1}+I_{d_{i}}} N(t)\left[\left(I R_{1 d_{i}}\right)\left(P e\left(t_{0}\right)\right)+\left(I R_{0 d_{i}}\right)\left(1-P e\left(t_{0}\right)\right)\right] \\
& \times \mathrm{dt}-\int_{t_{0}+I_{d_{i}}}^{t_{1}+I_{d_{i}}} N(t)\left[\left(I R_{1 d_{i}}\right) P e\left(t-I_{d_{i}}\right)\right. \\
& \left.+\left(I R_{0 d_{i}}\right)\left(1-P e\left(t-I_{d_{i}}\right)\right)\right] \mathrm{dt}
\end{aligned}
$$

The above formula can be further reduced to:
$\int_{t_{0}+I_{d_{i}}}^{t_{1}+I_{d_{i}}} N(t)\left[\left(I R_{1 d_{i}}-I R_{0 d_{i}}\right)\left(P e\left(t_{0}\right)-P e\left(t-I_{d_{i}}\right)\right)\right] \mathrm{dt}$

Let QAST $d_{i}$ denote the QAST of an average patient with disease $d_{i}$, QAST ${ }_{n}$ denote that of a normal person with the same age and gender. Then, one can obtain the total utility gained by the health prevention program by multiplying Eq. (2) with the expected utility lost per case for disease (event) $d_{i}$ (i.e. $\mathrm{QAST}_{n}-\mathrm{QAST}_{d_{i}}$ ). If one extends the preventive effect of reducing a particular exposure to $k$ number of age strata, in which each stratum $i$ has its own $I R_{1 d_{i}}$ and $I R_{0 d_{i}}$. Then, the total utility gained would be as follows:

$$
\begin{aligned}
& \sum_{i=1}^{k}\left\{\left(I R_{1 d_{i}}-I R_{0 d i}\right) \int_{t_{0}+I_{d_{i}}}^{t_{1}+I_{d_{i}}} N(t)\left[\left(P e\left(t_{0}\right)-P e\left(t-I_{d_{i}}\right)\right)\right] \mathrm{dt}\right\} \\
& \times\left(\mathrm{QAST}_{n}-\mathrm{QAST}_{d_{i}}\right)
\end{aligned}
$$

The above formula may also be considered as a summation of the preventive effect from reducing a specific exposure (e.g. smoking), which can result in several diseases (e.g. lung cancer, myocardial infarction, etc.) But the overlapping cases (i.e. persons afflicted with two or more diseases) must be discounted to avoid double counting. If the preventive program is also effective in reducing the severity of illness $d_{i}$, prolonging survival and improving the HRQL for cases who have taken preventive action, then the above equation should be added with the following term:

Table 4

Detailed calculations for estimation of the QALYs gained by enforcement of the helmet law for 1 year

No. of residents in Taipei

Incidence rate of head injury in Taipei (Chiu et al., 1996)

Proportion of traffic injuries among head injuries (Chiu et al., 1996)

Proportion of motorcycle injuries among traffic injuries (Chiu et al., 1996)

Incidence rate of head injury among motorcyclists $\left(=18.2 \times 10^{-4} \times 0.646 \times 0.80\right)$

Odds ratio of head injury for cyclists (Tsai et al., 1996)

With no helmet

With full face helmet

With partial coverage helmet

Proportion of cyclists with no helmet before intervention (Tsai et al., 1996)

Proportion of cyclists with full face helmet (Tsai et al., 1996)

Proportion of cyclists with partial coverage helmet (Tsai et al., 1996)

Incidence rate of head injury for cyclists with no helmet $(x)$

$\left(0.88 x+0.31 \times 0.085 x+0.73 \times 0.034 x=9.41 \times 10^{-4}\right)$

Proportion of cyclists with no helmet after execution of the law was reduced to 0.05

Proportion of full face helmet

Proportion of partial coverage helmet

Number of cases expected before regulation in 1 year

Number of cases expected after execution of law in 1 year

Average loss of expected quality-adjusted survival time of one case 4.8 QALYs

Total utility gained from the execution of helmet law in 1 year

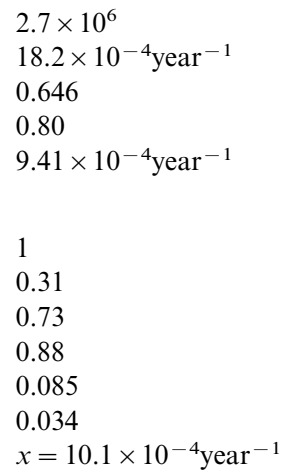

$(1-0.05) \times[0.085 /(0.085+0.034)]=0.673$

$(1-0.05) \times[0.034 /(0.085+0.034)]=0.269$

$2.7 \times 10^{6} \times 9.41 \times 10^{-4}\left(\right.$ year $\left.^{-1}\right) \times 1$ year $=2541$

$7 \times 10^{6} \times 10.1 \times 10^{-4} \times(0.05+0.673 \times 0.31+$

$0.269 \times 0.73)=1241$

$4.8 \times(2541-1241)=6240$ QALYs 
Table 5

Frequency and mean length of stay (LOS) in hospitals of subjects with different outcomes, stratified by helmet wearing states ${ }^{\mathrm{a}}$

Helmet wearing

\begin{tabular}{lllll}
\hline Yes $\left(n_{1}=28\right)$ & & No $\left(n_{2}=1332\right)$ & \\
\cline { 1 - 1 } No. of patients & LOS* & & No. of patients & LOS*
\end{tabular}

Glascow outcome scale

1 (dead)

2 (vegetative)

3 (disabled)

4 (independent but with sequel)

5 (complete recovery)

0
0
0
0
28

$\begin{array}{lrr}0 & 87 & 7.8 \pm 15.4 \\ 0 & 2 & 85.6 \pm 25.8 \\ 0 & 13 & 48.5 \pm 40.2 \\ 0 & 21 & 31.7 \pm 43.5 \\ 14.3 \pm 21.6 & 1209 & 17.2 \pm 23.7\end{array}$

${ }^{a} P=0.05$, student's $t$-test between the LOS of cases with helmet and LOS of surviving cases with no helmet.

$$
\begin{aligned}
& \sum_{i=1}^{k}\left\{\int_{t_{0}+I_{d_{i}}}^{t_{1}+I_{d_{i}}} N(t)\left[\left(I R_{0 d_{i}}\right)\left(P e\left(t_{0}\right)-P e\left(t-I_{d_{i}}\right)\right)\right] \mathrm{dt}\right\} \\
& \times\left(\mathrm{QAST}_{d_{i} p}-\mathrm{QAST}_{d_{i}}\right)
\end{aligned}
$$

where, $\mathrm{QAST}_{d_{i} p}$, the average QAST of patients who took preventive action but developed the illness $d_{i}$; $\mathrm{QAST}_{d_{i} p}-\mathrm{QAST}_{d_{i}}$, the expected difference of QAST between a non-exposed patient who was converted by the prevention program before the onset of illness and an average patient.

By combining Eqs. (5) and (6) together, the following general equation is obtained for the total expected utility gained from a preventive program in a population $N(t)$ with $k$ age strata

$$
\begin{aligned}
& \sum_{i=1}^{k} \int_{t_{0}+I_{d_{i}}}^{t_{1}+I_{d_{i}}}\left\{N(t)\left[\left(I R_{1 d_{i}}\right)\left(P e\left(t_{0}\right)-P e\left(t-I_{d_{i}}\right)\right)\right] \mathrm{dt}\right\} \\
& \times\left(\mathrm{QAST}_{n}-\mathrm{QAST}_{d_{i}}\right) \\
& -\sum_{i=1}^{k} \int_{t_{0}+I_{d_{i}}}^{t_{1}+I_{d_{i}}}\left\{N(t)\left[\left(I R_{0 d_{i}}\right)\left(P e\left(t_{0}\right)-P e\left(t-I_{d_{i}}\right)\right)\right] \mathrm{dt}\right\} \\
& \times\left(\mathrm{QAST}_{n}-\mathrm{QAST}_{d_{i} p}\right)
\end{aligned}
$$

\section{References}

Bergner, M., Bobbitt, R.A., Carter, W.B., Gilson, B.S., 1981. The sickness impact profile: development and final revision of a health status measurement. Medical Care 19, 787-805.

Chiu, W.T., Raporte, R.E., Gururaj, G., Raja, I.A., Pentel'enyi, T.J., Bouyoucef, K.A., Levy, A., Hung, C.C., 1996. Head injury in developing countries. In: Narayan, R.K., Wilberger, J.E., Povlishock, J.T. (Eds.), Neutrauma. McGraw-Hill, New York, pp. $905-911$.

Drummond, M.F., Stoddart, G.L., Torrance, G.W., 1987. Methods for the Economic Evaluation of Health Care Programmes, 1st. Oxford University, Oxford, pp. 5-17.

Euroqol group, 1990. Euroqol: a new facility for the measurement of health-related quality of life. Health Policy 16, 199-208.

Ghana Health Assessment Project Team, 1981. A quantitative method of assessing the heatlh impact of different diseases in less developed countries. International Journal of Epidemiology 10, $73-80$.
Glasziou, P.P., Simes, R.J., Gelber, R.D., 1990. Quality adjusted survival analysis. Statistical Medicine 9, 1259-1276.

Gold, M.R., Siegel, J.E., Russell, L.B., Weistein, M.C., 1996. CostEffectiveness in Health and Medicine, 1st. Oxford University, Oxford, pp. 98-106.

Hunt, S.M., McEwen, J., McKenna, S.P., 1986. Measuring Health Status. Beckenham, Croom Helm.

Hwang, J.S., Tsauo, J.Y., Wang, J.D., 1996. Estimation of expected quality adjusted survival by cross-sectional survey. Statistical Medicine 15, 93-102.

Kaplan, R.M., Bush, J.W., 1982. Health-related quality of life measurement for evaluation research and policy analysis. Health Psychology 1, 61-80.

LaPuma, J.L., Laqlor, E.F., 1990. Quality-adjusted life-year: ethical implications for physicians and policy makers. Journal of the American Medical Association 263, 2917-2921.

Lee, E.T., 1992. Statistical Methods for Survival Data Analysis, 2nd. Wiley, New York, p. 87.

MacKenzie, E.J., Damiano, A., Miller, T., Luchter, S., 1996. The development of the functional capacity index. Journal of Trauma 41, 799-807.

Miller, T.R., 1993. Costs and functional consequences of US roadway crashes. Accident Analysis and Prevention 25, 593-607.

Ministry of the Interior, Republic of China, 1989-1993. Abridged life tables of Taiwan-Fuchien area. Taipei, Taiwan, Ministry of the Interior.

Ministry of the Interior, Republic of China, 1994. Taiwan-Fukien Demographic Fact Book. Taipei, Taiwan, Ministry of the Interior.

Morrow, R.H., Bryant, J.H., 1995. Health policy approaches to measuring and valuing human life: conceptual and ethical issues. American Journal of Public Health 85, 1356-1360.

Murray, C.J.L., 1994. Quantifying the burden of disease: the technical basis for disability adjusted life years. Bulletin for the World Health Organisation 72, 429-445.

Patrick, D.L., Erickson, P., 1993. Health Status and Health Policy: Quality of Life in Health Care Evaluation and Resource Allocation. Oxford University, Oxford, pp. 9-12.

Robinson, R., 1993. Cost-utility analysis. British Medical Journal 307, 859-862.

Rosser, R., Cottee, M., Rabin, R., et al., 1992. Index of health-related quality of life. In: Hopkins, A. (Ed.), Measures of Quality of Life. Royal College of Physicians of London, London, pp. 81-90, $147-153$.

Rubinstein, R.Y., 1981. Simulation and the Monte Carlo Method. Wiley, New York.

Sorkin, A.L., 1992. Health Economics, 3rd. Lexington Books, An Imprint of Macmillan, New York, pp. 10-13. 
Stat Sci Division, MathSoft Inc., 1995. S-plus guide to statistical and mathematical analysis, version 3.3. Seattle, Washington, MathSoft Inc.

Taiwan Provincial Institute of Family Planning, Population Studies Center, 1989. University of Michigan, Institute of Gerontology, University of Michigan. 1989 survey of health and living status of the elderly in Taiwan: questionnaire and survey design. Comparative study of the elderly in four Asian countries, research report No. 1.

Torrance, G.W., 1986. Measurement of health state utilities for economic appraisal: a review. Journal of Health Economics 5, 1-30.

Tsai, Y.J., Wang, J.D., Huang, W.F., 1996. A case-control study on the effectiveness of prevention of head injuries by helmet among motorcycle riders in Taipei. American Journal of Epidemiology 142, 974-981.

Ware, J.E., Sherbourne, C.D., 1992. The MOS 36-item short-form health survey (SF-36): I. Conceptual framework and item selection. Medical Care 30, 473-483.

US Department of Transportation, National Highway Traffic Safety Administration, 1998. Further Analysis of Motorcycle Helmet Effectiveness Using CODES Linked Data. Research Note U.S. Department of Transportation, National Highway Traffic Safety Administration.

Watson, G.S., 1966. Smooth regression analysis. Sankha, Series A 26, $359-378$ 